\title{
Resource Allocation in D2D Communication in Cellular Mode
}

\author{
Khoa Anh TRAN ${ }^{1, *}$ \\ ${ }^{1}$ Department of Electronics and Telecommunication Engineering, \\ Faculty of Electrical and Electronics Engineering, \\ Ton Duc Thang University, Ho Chi Minh city, Vietnam \\ *Corresponding Author: Khoa Anh TRAN (email: trananhkhoa@tdtu.edu.vn) \\ (Received: 08-August-2018; accepted: 26-October-2018; published: 31-October-2018) \\ DOI: http://dx.doi.org/10.25073/jaec.201823.198
}

\begin{abstract}
Device-to-Device (D2D) has attracted substantial research attention recently and has been recognized as an essential approach to performance improvement in $5 G$ networks, due to its potential to improve coverage, spectrum efficiency, and energy within the existing cellular network. In this paper, we refer to an LTE-A scenario in which the underlay mode is adopted to allow D2D pairs to communicate directly by sharing sub-channels with Cellular Users (CUEs) and cellular mode (CELLM), where two D2D users communicate through the eNB as conventional CUEs and no direct D2D link is established. In this case, the eNB is used as a relay. Our aim is to propose heuristic resource allocation schemes to distribute radio resources among CUEs and D2Ds in a cell taking the interference because of pairing into account. Finally, an analytical approach is proposed to characterize CUE and D2D capacity as well as outage probability for D2D cellular mode.
\end{abstract}

\section{Keywords}

Resource Allocation, Device-to-Device Communication, Cellular Mode, Underlay Mode.

\section{INTRODUCTION}

The increasing popularity of mobile devices with data-hungry applications has resulted in a fast-growing demand for high-rate data access experiences. It will be challenging for the currently-deployed Long Term Evolution - Advanced (LTE-A) systems to satisfy such demand in the future. One promising solution is represented by Device-to-Device (D2D) communications, in which nearby users can setup a direct communication link to transmit data to each other without going through the base station (eNB) [1. In a recent report by the Third-Generation Partnership Project (3GPP), D2D communications have been considered as a promising technique in Long-Term Evolution Advanced (LTE-Advanced) standard since Release 12 [2, 3] and have many applications, such as Machine-to-Machine (M2M) and Vehicle-toVehicle (V2V) communications, social discovery, proximity-based services, coverage extension, traffic offloading, and public safety.

There are basically three different communication modes for D2Ds: cellular mode, overlay mode, and underlay mode [4. In the cellular mode, the D2D pair transmit via the eNB (a link from D2D Transmitter, D2D Tx, to eNB and another link from eNB to D2D Receiver, D2D $\mathrm{Rx}$ ) using resources just like traditional Cellular Users (CUEs). However, the network 
spectrum utilization can be boosted only if direct communications are allowed between D2D Tx and D2D Rx using either a distinct portion of the spectrum (overlay mode) or even better the same spectrum (underlay mode) as that for CUEs. The analytical framework developed in [5] uses tools from stochastic geometry [20] and accounts for power control, maximum transmit power of the CUEs, and mode selection of CUEs in an uplink cellular network. In [6], this paper presents a framework based on a probabilistic distance and path-loss model to obtain the distributions of signal, interference, and further Signal-to-Interference-plus-Noise Ratio (SINR), based on which, the performance metrics that are functions of SINR-such as outage probability and capacity-can be analyzed. However, they do not provide the analytical framework to compare with the D2D in cellular mode. In [7, the authors present a simple, interesting geometric interpretation of the physical implications of admission conditions and others. A distance-constrained resource-sharing method is proposed to select the CUE for a given D2D pair to share it the sub-channel, and outage performance of D2D communication is also proposed. The aim of this method is to mitigate the interference from the cellular transmissions to the D2D pair. The comparison of outage probability analysis and numerical are evaluation reveal that there is an optimal minimum distance between the D2D receiver and its paired cellular UE, and the advantage of this method significantly reduces the outage probability of D2D communications in [8. In view of these findings, in this paper, we refer to the underlay mode that poses new challenges for managing the interference caused by the resource sharing between D2D pairs and CUEs and develop a new framework that can help network planners to effectively tune the network parameters, and thus achieve the optimum system performance for cellular D2D communications.

The major contributions of this paper are summarized as below:

- We study the SINR model and resource allocation for D2D enabled cellular networks.
- A new analytical approach is proposed to characterize CUE and D2D capacity as well as outage probability for cellular mode.

The rest of the manuscript is structured as follows. We describe the system model in Section 2. Section 3 presents the problem formulation of CUE and D2D capacity and outage probability analysis in cellular mode. We present our numerical results in Section 4 before our concluding remarks in Section 5.

\section{SYSTEM MODEL AND PROBLEM FORMULATION}

This section first introduces our system model for the D2D cellular mode in LTE-A system, SINR model and basic scheduling approaches. The QoS requirements are then specified in terms of outage probability.

\subsection{Assumptions}

Our scenario consists of a single-cell system, where eNBs are located at cell centers, and users are uniform scatter in the cells. The cellular layout is hexagonal regular with eNBs at the cell center. There are two types of users in the network, namely CUEs and D2Ds pairs (i.e., D2D Tx and D2D Rx). CUEs are primary users of cellular radio resources.

We refer here to the uplink bandwidth for CUEs transmissions to the eNB that also used for D2D communication ${ }^{1}$. The available spectrum is divided into $N$ orthogonal sub-channels. Assuming each sub-channel can be used by both CUEs and D2D pairs. For the sake of simplicity, we assume that each sub-channel allocated to a CUE can be shared by (maximum) one D2D pair of the same cell in the underlaying mode. We consider a reference eNB denoted by letter $C$,

\footnotetext{
${ }^{1}$ We consider the use of the uplink bandwidth for D2D underlay communications because of the asymmetric use of uplink and downlink frequencies and because D2D communications are only causing interference to the eNB. The SINR model in underlay mode of this paper, which is proposed in EUCNC 2017 [9] and ICC 2018 [10].
} 
containing $M$ CUEs and $K$ D2D pairs randomly distributed. The sets of CUEs and D2D pairs in the cell are denoted by $\mathcal{M}=\{1,2, \ldots, M\}$ and $\mathcal{K}=\{1,2, \ldots, K\}$, respectively. Let $p_{m, n}, p_{k, n}$, and $p_{c, n}$ be the transmission powers of CUE $m$, D2D Tx $k$, and eNB $C$ on sub-channel $n$, respectively. $N_{0}$ and $B$ are the background noise power spectral density at ambient temperature and the sub-channel bandwidth, respectively.

The channel power gains of the different links are determined by the path loss models and are used to determine the SINR, as described in the next sub-Section. The Stanford University Interim (SUI) path loss model [11] and the WINNER II path loss model [12] are adopted in this paper as follows: SUI path loss used for the communication links between CUEs and eNB, between D2Ds and CUEs, between D2Ds of different cells; WINNER II path loss is used for the communication links between D2D pairs having direct communication inside the cell. We include shadowing margin terms in the path loss models to take the obstacles to signal propagation into account.

Resource allocations are performed on a TTI (Transmission Time Interval) basis, where TTI $=1 \mathrm{~ms}$ in LTE.

\subsection{SINR model for cellular mode}

In the cellular mode, two D2D users communicate through the eNB as conventional CUEs and no direct D2D link is established. In this case, the eNB is used as a relay. The D2D pairs in cellular mode perform like familiar CUEs. We assume necessarily that any other D2D pair and CUE do not use reuse their sub-channels in the same cell. Of course, D2Ds and CUEs use the same transmission power. In this mode, D2Ds and CUEs are all together in the same group, and for both of them, we have the same SINR conditions. In particular, SINR for CUE $m$ (as well as for D2D Tx $k$ ) at eNB $C$ on sub-channel $n$ can be written as:

$$
\operatorname{SINR}_{m, n}^{C \text { or } D, U L}=\frac{p_{m, n}\left|h_{m, n}\right|^{2}}{N_{0} B}
$$

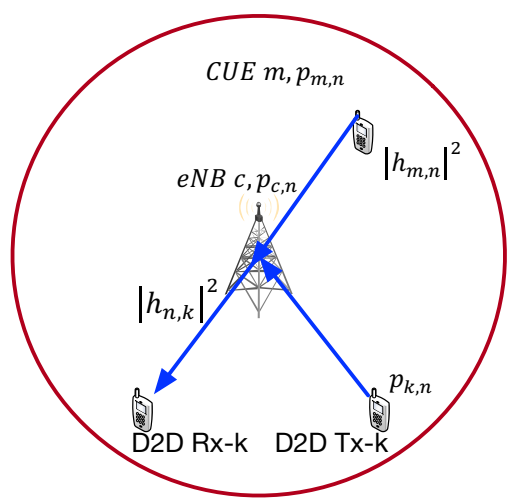

Fig. 1: Illustration of Device-to-Device cellular mode, showing the channel gains and the transmission powers of the different links

In the D2D case, we also need a downlink transmission from the eNB to D2D Rx $k$. Hence, the SINR in downlink on sub-channel $n$ (downlink) can be written as:

$$
\operatorname{SINR}_{k, n}^{D, D L}=\frac{p_{c, n}\left|h_{n, k}\right|^{2}}{N_{0} B}
$$

where

- $\left|h_{n, k}\right|^{2}$ and $\left|h_{m, n}\right|^{2}$ are defined in the same way;

- $N_{0}=174 \mathrm{dBm} / \mathrm{Hz}$ and $B=180 \mathrm{kHz}$ are the background noise power spectral density at ambient temperature and the subchannel bandwidth, respectively.

Then, we consider that the capacity for the $k^{t h}$ D2D pair depends on the minimum of the capacities of UL and DL (the D2D link, in this case, is obtained as the cascade of UL and DL) that in turn depend on the respective SINR values, according to (1) and (2), using the modified Shannon capacity formula in (3).

The capacity of a given sub-channel $n, C_{n}$, can be computed according to the SINR value on the basis of the modified Shannon formula [9] as:

$$
C_{n}=\left\{\begin{array}{l}
B \times \log _{2}\left(1+\frac{S I N R_{n}}{\Gamma}\right), \text { if } S I N R_{n}> \\
S I N R_{\min } \\
0, \quad \text { if } S I N R_{n}<S I N R_{\min }
\end{array}\right.
$$


where $\Gamma=-\frac{2}{3} \ln (5 \times B E R)$, being $\Gamma$ a constant and $B E R=0.00005$. The pairing of D2Ds and CUEs causes some mutual interference that may lead the CUE and/or the D2D in outage conditions, when the received SINR is below the $S I N R_{\text {min }}$ threshold value $(=-2.1054 \mathrm{~dB}$ in our numerical evaluations).

\subsection{Basic scheduling approaches}

In underlay mode, the resource management algorithm is divided into two phases. In the first phase, we select CUEs to be serviced in the current TTI at random, and then we assign sub-channels to these CUEs according to a random mapping 2 . Secondly, we also select D2D pairs to be serviced in the current TTI at random and then we assign the subchannels to these D2D pairs according to a random mapping (underlay mode). Finally, we apply the SINR model to calculate the capacity and outage probability for underlay mode and next Section we propose a new framework to calculate the CUE and D2D capacity and outage probability analysis. Moreover, Algorithm 1 proposes how to assign the resource (sub-channel) for CUE and calculate the CUE capacity in the simulation (CELLM case).

\section{CUE AND D2D CAPACITY AND OUTAGE PROBABILITY ANALYSIS IN CELLULAR MODE}

In this section, we propose a novel analytical approach for both capacity and outage probability prediction for CUEs and D2Ds based on an SINR model (single-cell case) and a random

\footnotetext{
${ }^{2}$ The meaning of random mapping is defined by assigning the sub-channel for each CUE and D2D pair random sub-channel. Note that, in one TTI, the sub-channel only can be used for one CUE and D2D pair.
}

Algorithm 1 Pseudo code to calculate the CUE capacity for UL and DL in the simulation.

Input: $\mathcal{M}, N, T T I s, S I N R_{\text {min }}, P_{t x e N B}, P_{t x C U E}$. Output: CUE and D2D capacity, and outage probability.

Step 1: We select CUEs to be serviced in the current TTI on the basis of Round Robin scheduling (i.e., cyclic ordering) schemes; resources are here assigned to the selected CUEs on the basis of random mapping.

Step 2: We calculate the SINR and capacity of CUEs for both UL and DL as the algorithm, which is presented below

1: procedure SUB-CHANNEL ALLOCATION (,)

\section{2: for $m \in M$ do}

3: Calculate the $S I N R_{C U E}^{U L}(m)$ of CUE in UL by using (1).

4: Calculate the $S I N R_{C U E}^{D L}(m)$ of CUE in DL by using $(2)$.

5: Calculate the $C U E^{U L}(m)$ capacity and outage probability for UL by using (3), and set $C U E^{U L}(m)$ is a CUE capacity for UL. Calculate the $C U E^{D L}(m)$ capacity and outage probability for DL by using (3). Save it as a vector $\left[C U E^{U L}(m), C U E^{D L}(m)\right]$.

6: Select $\min \left(C U E^{U L}(m), C U E^{D L}(m)\right)$ set it is a D2D capacity for cellular mode.

uniform distribution of CUEs and D2Ds cellular mode.

\subsection{CUE and D2D capacity analysis in cellular mode}

Also in the cellular mode, we study the performance of a single cell (no inter-cell interference). D2D users and CUEs are all together in the same group and experience the same SINR condition. Let us consider a generic CUE at distance $a \in[0, R]$ from the eNB of the reference cell (having the eNB in the origin) with radius 


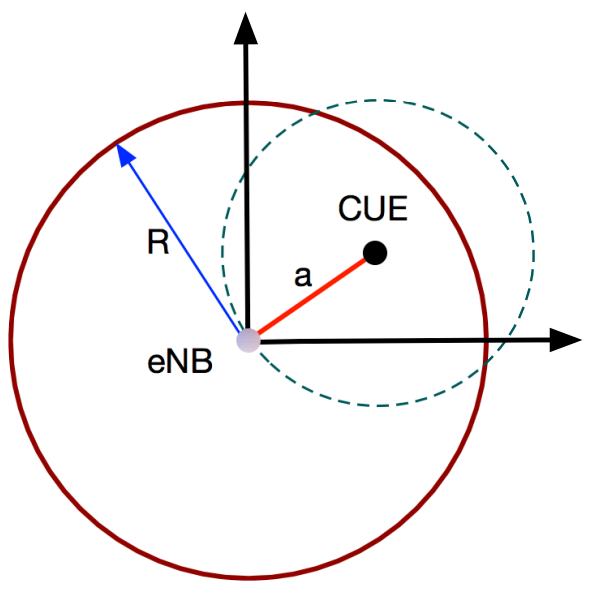

Fig. 2: CUE capacity model for circular cells.

$R$. The SINR values of this CUE in UL and DL can be calculated as:

$$
\begin{aligned}
& \operatorname{SINR}_{C U E}^{U L}(a)=\frac{\frac{P_{T x} C U E}{\text { pathloss }(a)}}{N_{0} B}, \\
& \operatorname{SINR}_{C U E}^{D L}(a)=\frac{\frac{P_{T x} e N B / S}{\text { pathloss }(a)}}{N_{0} B},
\end{aligned}
$$

where

- $P_{T x} e N B$ is the total transmission power of $\mathrm{eNB} c$;

- $S$ is the number of sub-channels in a cell;

- pathloss $(a)$ is the path loss in linear scale of the communication link from CUE to eNB and vice versa.

Analogously to (3), the capacity available for a generic CUE in UL and DL can be expressed as (when we neglect the $S I N R_{\text {min }}$ threshold effects for the capacity derivation):

$$
\begin{aligned}
& C_{C U E}^{U L(D L)}(a)= \\
& S \times B \times \log _{2}\left(1+\frac{S I N R_{C U E}^{U L(D L)}(a)}{\Gamma}\right),
\end{aligned}
$$

We have to remove the conditioning on $a$ considering its distribution that is obtained by taking the circular symmetry of the problem into account as follows:

$$
p d f(a)=\frac{2 \pi a d a}{\pi R^{2}}
$$

Then, we can express the total cell capacity for UL or DL as follows:

$$
\begin{gathered}
T_{U L(D L)}=\int_{0}^{R} C_{C U E}^{U L(D L)}(a) \times\left(\frac{2 a d a}{R^{2}}\right) \\
=\frac{2 S B}{R^{2}} \int_{0}^{R} a \\
\times \log _{2}\left(1+\frac{S I N R_{C U E}^{U L(D L)}(a)}{\Gamma}\right) d a .
\end{gathered}
$$

where we neglect outage effects.

Then, in uplink the total CUE capacity (one cell) can be calculated as:

$$
C U E_{\text {capacity }}=\frac{M}{(M+K)} \times T_{U L} .
$$

Moreover, the total D2D capacity (one cell) can be expressed as:

$$
\begin{aligned}
& D 2 D_{\text {capacity }}=\left(\frac{2}{R^{2}}\right) \times \\
& \frac{K}{(M+K)} \int_{0}^{R} \min \left(\left(C_{C U E}^{U L}(a), C_{C U E}^{D L}(a)\right)\right) a d a .
\end{aligned}
$$

These capacity formulas are valid considering that the cell is saturated, so that $M+K \geq S$, the number of sub-channels of the cell. Next subsection, we would like to propose the CUE and D2D outage probability analysis in cellular mode. 


\subsection{CUE and D2D outage probability analysis in cellular mode}

Let us consider a generic CUE at distance $a \in$ $[0, R]$ from the eNB, which is inside the reference cell (having the eNB in the origin) with radius $R$. Referring to Fig. 2, the following formula is used to characterize the maximum distance $a, a_{\max }$ after which the CUE in the cell experiences outage conditions depending on threshold $S I N R_{\text {min }}$ (here expressed in $\mathrm{dBs}$ ):

$$
\frac{\frac{P_{T x} C U E}{p a t h l o s s\left(a_{\max }\right)}}{N_{0} B}=10^{\frac{\operatorname{SINR}_{\min }}{10}}
$$

where pathloss $\left(a_{\max }\right)=\operatorname{SUI}\left(a_{\max }\right)$.

With some algebraic manipulations, from (9) we obtain the following formula:

$$
S U I\left(a_{\max }\right)=\frac{P_{T x} C U E}{N_{0} B} \times 10^{\frac{-S I N R_{\min }}{10}} .
$$

For the sake of simplicity, let us assume that $a_{\max }>R_{0}$ of the SUI model so that (11) can be solved with respect to $a_{\max }$ as follows:

$$
\begin{aligned}
a_{\max } & =\sqrt[\gamma]{\psi^{-1} \times \omega^{-\gamma} \times 10^{\frac{-\phi}{10}}} \\
& \times \sqrt[\gamma]{\left(\frac{P_{T x} C U E}{N_{0}} \times 10^{\frac{-S I N R_{\min }}{10}}\right)},
\end{aligned}
$$

and where terms $\phi, \psi$, and $\omega$ can be expressed as follows according to the SUI path loss model in [7.

The CUE outage probability is determined considering that all CUEs with distance $a$ from $a_{\max }$ to $R$ are in outage conditions. Considering the circular symmetry of the problem, then this probability can be expressed differentiating the case $a_{\max } \leq R$ from the case $a_{\max }>R$. If $a_{\max }$ $\leq R$, we have:

Otherwise, if $a_{\max }>R$, the CUE outage probability is equal to 1 . Then, the CUE outage probability can be expressed as follows:

$$
P_{\text {CUEoutage }}=\left\{\begin{array}{l}
1-\left(\frac{a_{\max }}{R}\right)^{2}, \quad \text { if } a_{\max } \leq R \\
0, \quad \text { if } a_{\max }>R .
\end{array}\right.
$$

Let us now consider the D2D outage probability. Let us denote: $P_{O U L}$ $=U L$ outage probability and $P_{O D L}=$ $D L$ outage probability, where $P_{O U L}$ is given by (13) and $P_{O D L}$ is obtained by adapting (13) to the downlink case, where $a_{\max }$ is obtained using $S I N R_{D L}$ and considering the eNB transmission power divided among $S=\frac{N}{F}$ sub-channels of the cell.

The D2D outage probability can be computed considering the combination of UL and DL effects as shown in (14). In particular, we characterize the probability of no D2D outage as the product of the probability of no UL outage times the product of no DL outage being these two as independent events. Then, we have:

$$
\begin{gathered}
P_{D 2 \text { Doutage }}=1-\left(1-P_{O U L}\right) \times\left(1-P_{O D L}\right) \\
=P_{O U L}+P_{O D L}-P_{O U L} \times P_{O D L} .
\end{gathered}
$$

This formula can also be justified by means of the probability of the union of non-disjoint events $\mathrm{A}$ and $\mathrm{B}: \mathrm{P}(\mathrm{A} \cup \mathrm{B})=\mathrm{P}(\mathrm{A})+\mathrm{P}(\mathrm{B})-$ $\mathrm{P}(\mathrm{A} \cap \mathrm{B})$, where $\mathrm{P}(\mathrm{A} \cap \mathrm{B})=\mathrm{P}(\mathrm{A}) \mathrm{P}(\mathrm{B})$ since these events are independent.

\subsection{Average cell capacity and outage probability in cellular mode}

In this mode, the scheduler determines the CUE $m^{*}$ of the reference cell that is allocated on sub-channel $n$ in TTI and the corresponding SINR values in UL. Then, the capacity of a D2D pair depends on the minimum of the capacities of UL and DL (the D2D link, in this case, is obtained as the cascade of UL and DL) that in turn depend on the respective SINR values. We sum over all sub-channels of the cell and overall the TTIs in the simulation, and then we divide by the simulation length in TTIs in order to achieve 
the average capacity from simulations. Then, the average (total) cell capacity in uplink can be computed as follows: where

- $M$ is the number of CUEs in the cell;

- $K$ is the number of D2D pairs in the cell;

- $\frac{M}{(M+K)}$ is the weight of CUEs over the total number of users (CUEs plus D2D pairs) in the system;

- $\frac{K}{(M+K)}$ is the weight of D2D pairs over the total number of users (CUEs plus D2D pairs) in the system.

We apply the coefficients $\frac{M}{(M+K)}$ and $\frac{K}{(M+K)}$ to determine the average cell capacity in (15), because in the cellular mode (CELLM), one channel can be only rigidly assigned to a CUE or to a D2D.

In the cellular mode $(C E L L M)$, the outage probability for CUEs and D2Ds has been estimated from simulations according to the approaches by equations (16) and (17).

\section{NUMERICAL RESULTS}

\subsection{Simulation settings}

We have implemented a Matlab simulator to compare D2D communication underlay mode and cellular mode. We repeat 20 times each simulation run of 2000 TTIs with different randomized CUE and D2D pair positions and shadowing margin (Monte Carlo approach). The settings of the simulator are provided in Table 1. Results are obtained only from a central (reference) cell, even if we also simulate adjacent cells to achieve realistic interference conditions.

In our simulations, CUEs and D2D Txs are uniformly distributed in the cells that have a circular shape with eNB at the center. CUEs and D2D Txs positions are determined as uniformly distributed inside circularly-shaped cells with radius $R$. The positions uniformly distributed in the circle can be characterized in polar coordinates $(r, \theta)$ according to the following
Tab. 1: Simulator settings.

\begin{tabular}{|c|c|}
\hline System parameters & Values \\
\hline Cell radius (uplink), $R$ & $500 \mathrm{~m}$ \\
\hline System bandwidth & $10 \mathrm{MHz}$ \\
\hline $\begin{array}{l}\text { Transmission power of } \\
\text { an eNB, } P t x_{e N B}\end{array}$ & $46 \mathrm{dBm}$ \\
\hline $\begin{array}{l}\text { Transmission power of } \\
\text { an CUE, } P t x_{C U E}\end{array}$ & $24 \mathrm{dBm}$ \\
\hline $\begin{array}{l}\text { Transmission power of } \\
\text { an D2D, } P t x_{D 2 D}\end{array}$ & $10-24 \mathrm{dBm}$ \\
\hline $\begin{array}{l}\text { Background noise power } \\
\text { spectral density, } N_{0}\end{array}$ & $\begin{array}{l}-174 \\
\mathrm{dBm} / \mathrm{Hz}\end{array}$ \\
\hline $\begin{array}{l}\text { RB (sub-channel) band- } \\
\text { width, } B\end{array}$ & $180 \mathrm{kHz}$ \\
\hline $\begin{array}{l}\text { Number of sub-channels, } \\
N\end{array}$ & 50 \\
\hline $\begin{array}{l}\text { Length of a simulation } \\
\text { run in } \# T T I s\end{array}$ & 2000 \\
\hline $\begin{array}{l}\text { Number of cells simu- } \\
\text { lated }\end{array}$ & 1 \\
\hline $\begin{array}{l}\text { Number of CUEs and } \\
\text { D2D }\end{array}$ & 50 \\
\hline $\begin{array}{l}\text { Min and Max distance } \\
\text { between D2D Tx and } \\
\text { D2D Rx, } L_{1} \text { and } L_{2}\end{array}$ & $0 \mathrm{~m}$ and $60 \mathrm{~m}$ \\
\hline $\begin{array}{l}\text { Outage threshold, } \\
S I N R_{\min }\end{array}$ & $-2.1054 \mathrm{~dB}$ \\
\hline Shadowing margins & $\begin{array}{lll}16 & \mathrm{~dB} & \text { for }\end{array}$ \\
\hline $\begin{array}{l}S_{C U E} \text { and } S_{D 2 D} \text { (path } \\
\text { loss) }\end{array}$ & $\begin{array}{l}\text { CUEs and } \\
5 \mathrm{~dB} \text { for } \mathrm{D} 2 \mathrm{D} \\
\text { links }\end{array}$ \\
\hline
\end{tabular}

pdfs of independent variables:

$$
p d f(r)=\frac{2}{R^{2}} r, \quad p d f(\theta)=\frac{1}{2 \pi},
$$

where $r \in[0, R]$ and $\theta \in[0,2 \pi]$. Moreover, for each D2D Tx, we have to determine the position of the corresponding D2D Rx considering that the D2D Rx is uniformly distributed around the D2D Tx in a ring within a distance from $L_{1}$ to $L_{2}$. Then, taking the D2D Tx position as a reference now, we determine the relative position of the $\mathrm{D} 2 \mathrm{D} \mathrm{Rx}$ in polar coordinates $(D, \varphi)$ as:

$$
p d f(D)=\frac{2}{L_{2}^{2}-L_{1}^{2}} D, \quad p d f(\varphi)=\frac{1}{2 \pi},
$$

where $D \in\left[L_{1}, L_{2}\right]$ and $\varphi \in[0,2 \pi]$.

In each scheme for both of D2D underlay mode and cellular mode, firstly we apply the Algorithm 1 to select the CUE and D2D pairs to be served and assign sub-channel allocation, 
Average cell capacity $=S \times \frac{\left(\begin{array}{c}\sum_{t=1}^{\# T T I}\left(\frac{M}{M+K}\right) B \log _{2}\left(1+\frac{\operatorname{SIN}_{m^{*}, n}^{C, U L}(t)}{\Gamma}\right)+ \\ \sum_{t=1}^{\# T T I}\left(\frac{K}{M+K}\right) \operatorname{Bmin}\left[\log _{2}\left(1+\frac{\operatorname{SIN} R_{k^{*}, n}^{C, U L}(t)}{\Gamma}\right), \log _{2}\left(1+\frac{\operatorname{SIN}_{k^{*}, n}^{C D L}(t)}{\Gamma}\right)\right]\end{array}\right)}{\# \text { TTI }}$

OutageProb $C U E=\frac{\sum_{t=1}^{\# T T I} \frac{\text { Number of scheduled CUEs in outage condition in TTI } t}{\text { Total number ofCUEs allocated in TTI t }}}{\# T T I}$.

and determine capacity and outage conditions for both CUEs and D2D pairs. At each TTI, we determine the new capacity value and a new outage probability cases. Then, we take the average for the capacity and the outage probability for both CUEs and D2D pairs all over the runs.

\subsection{Analysis results and comparison with simulations}

In this paper, we show simulation results compared with analysis ones (according to Section $3 . \quad$ for the cellular mode $(C E L L M)$ and the underlaying case with a random scheduler and random pairing $\left(R n d^{2}\right)$, and the scheduled D2Ds are paired with the CUEs at the farthest distance $(R R-F A R)$ [9] in the simulation. We refer to the system settings in Table 1. In this study (single-cell case), cells have a circular shape in both analysis and simulation. In this scenario, the reuse factor is equal to 1 . The results are shown in Figs. 3 and 4 for what concerns capacity and outage probability as a function of the D2D transmission power level for 50 CUEs, 50 D2D pairs, and 50 subchannels. It can be observed that as the D2D power increases, the D2D capacity increases as well and the D2D outage probability decreases in the underlaying mode $R n d^{2}$ and $(R R-F A R)$. Especially, the D2D capacity in $(R R-F A R)$ is higher than the $\left(R n d^{2}\right)$, because we select the farthest distance of CUEs to share the subchannel with the D2D pairs, that means the interference of CUEs are smallest. Moreover,
CUE capacity also decreases and CUE outage increases because of the interference from D2D transmissions increases and are equivalent for all the algorithm in underlay mode $\left(R n d^{2}\right.$ and $R R-F A R)$. Note that the outage probability for CUEs in underlay mode with the simple $R n d^{2}$ and $(R R-F A R)$ scheme too high. We also present the capacity and outage probability of D2Ds and CUEs in cellular mode (CELLM), where CUE and D2D transmission powers are the same. In this case, the capacity values for CUEs and D2Ds increase with the transmission power. On the other hand, outage probabilities are zero, because there is no interference in the cellular mode (CELLM) single-cell scenario. In all the cases of Figs. 3 and 4 , there is a good agreement between analysis and simulation results for CELLM case. Moreover, from Fig. 3 We see that $R R-F A R$ is the best scheme in terms of capacity and outage probability for D2Ds.

We also analyze the capacity and outage probability of CUEs and D2Ds for CELLM cases as functions of the number of D2D pairs $K$, with $K$ increasing from 20 pairs to 70 pairs, 120 CUEs and 50 sub-channels $(N)$, and compare with the underlay mode $\left(R n d^{2}\right.$ and $\left.R R-F A R\right)$. Let us refer to Figs. 5 and 6 . It is interesting to note that the D2D capacity in underlay mode increases as the number of $\mathrm{D} 2 \mathrm{D}$ pairs grows since more D2D pairs have channels allocated and D2D outage probability increases as well. Besides, when $K \geq N$, D2D capacity, and D2D outage probability do not vary because the use of sub-channels by D2Ds is saturated. As 
OutageProb $\operatorname{Pr}_{D}=\frac{\sum_{t=1}^{\# T T I} \frac{D 2 D \text { pairs numbers in outage condition at } T T I t}{\text { Total number of D2D pairs allocated at TTI } t}}{\# T T I}$.
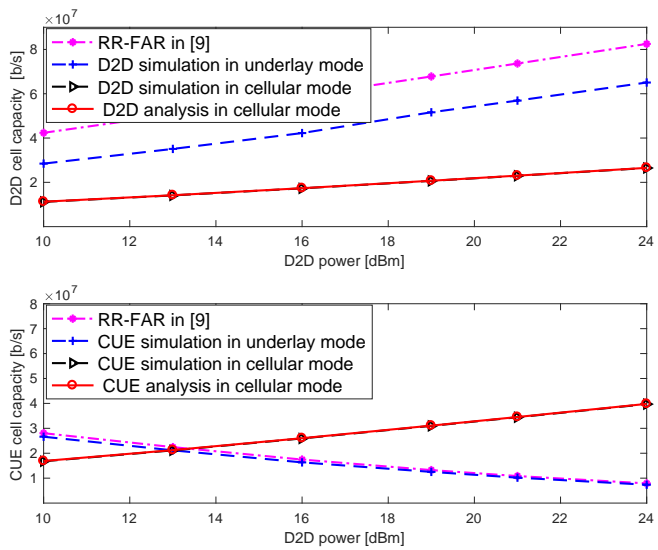

Fig. 3: D2D and CUE cell capacity as function of the D2D transmission powers in underlay mode scheme) and cellular mode (CELLM).
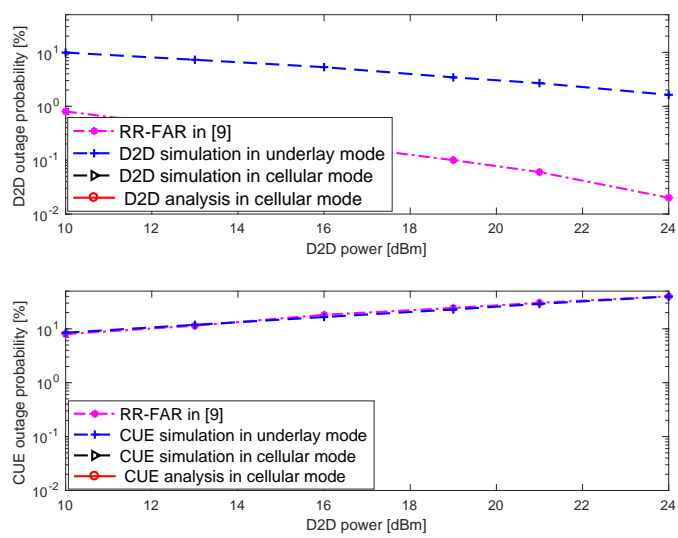

Fig. 4: D2D and CUE outage probability as function of the D2D transmission powers in underlay mode scheme) and cellular mode (CELLM).

for CUEs, capacity in underlay mode decreases and outage probability increases with the number of D2Ds. Moreover, CUE capacity and outage probability saturate when $K \geq N$.

Let us now comment the results for $C E L L M$ case in Figs. 5 and 6. We can see that D2D capacity increases with the number of $\mathrm{D} 2 \mathrm{D}$ pairs because according to $(\sqrt{9})$ this capacity is proportional to $\frac{K}{M+K}$, where the numerator $K$ is the D2D pair numbers, and the denominator $(M+K)$ is the total number of users (both CUEs and D2D pairs) in the system. On the other hand, CUE capacity decreases with the increase in the number of $\mathrm{D} 2 \mathrm{D}$ pairs, because on the basis of $(10)$ ), this capacity is proportional to $\frac{M}{(M+K)}$, where $M$ is the number of CUEs in the reference cell. Then, we have no outage probability for CUEs with CELLM case because there is no interference with a single cell and background noise has not impact because of the small cell range.

Unfortunately, with current settings and the use of the simple $R n d^{2}$ and $R R-F A R$ scheme the outage probability of the underlay case is too high.

Finally, we can see that there is good agreement between analysis and simulation results, thus cross-validating both the implemented simulator and the analytical approach for a cellular mode. And the advantages of D2D communication in underlay mode, since the capacity is higher than the traditional cellular network.

\section{CONCLUSION}

This paper presented an analytical framework based on a distance and path-loss model for the performance analysis in a cellular network with cellular D2D communications. Based on the obtained results, the interference and outage probabilities for both the cellular and D2D communications in an uplink reusing scenario are thoroughly investigated. The accurate analysis demonstrated the promising potentials of the proposed framework, which we believe is a significant complementary work to the existing approaches and results, and can provide meaningful insights and guidelines for the design and optimization of D2D communications 

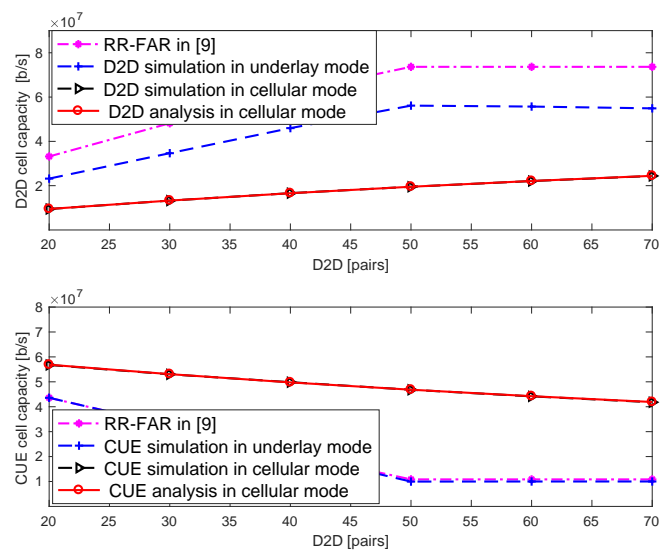

Fig. 5: D2D and CUE cell capacity as function of the number of D2D pairs in underlay mode scheme) and cellular mode $(C E L L M)$ for CUE (D2D) transmission power of $24 \mathrm{dBm}(21 \mathrm{dBm})$.

in the next-generation cellular networks. Based on the proposed framework, further investigation can be done for modeling and analyzing the uplink reusing scenario, the multi-cell scenarios, and considering other channel impairments such as log-normal shadowing and fast fading.

\section{References}

[1] Phunchongharn, P., Hossain, E., \& Kim, D. I. (2013). Resource allocation for device-todevice communications underlaying LTEadvanced networks. IEEE Wireless Communications, 20(4), 91-100.

[2] Lin, X., Andrews, J., Ghosh, A., \& Ratasuk, R. (2014). An overview of 3GPP device-to-device proximity services. IEEE Communications Magazine, 52(4), 40-48.

[3] 3GPP 23.703 (2013), Study on architecture enhancements to support Proximitybased Services (ProSe), Technical report (TR),Rel 12, v. 05.

[4] Yu, C., Doppler, K., Ribeiro, C., Tirkkonen, O. (2011). Resource Sharing Optimization for Device-to-Device Communication Underlaying Cellular Network. IEEE Transactions on Wireless Communications, 10(8), 2752-2763.
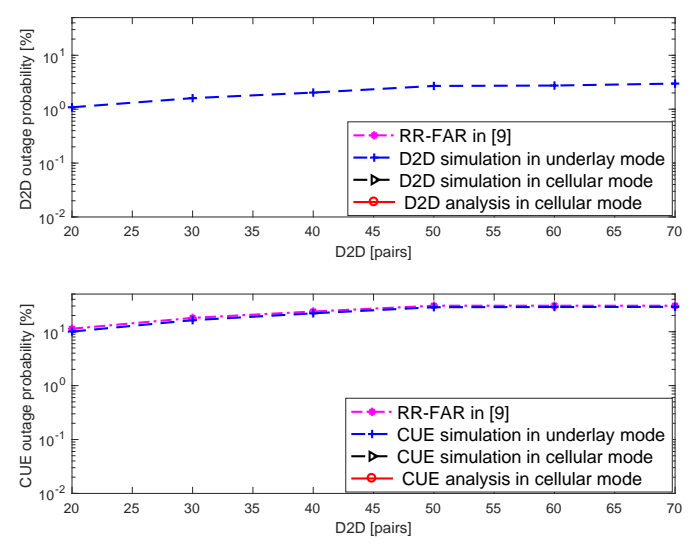

Fig. 6: D2D and CUE cell outage probability as function of the number of D2D pairs in underlay mode scheme) and cellular mode (CELLM) for CUE (D2D) transmission power of $24 \mathrm{dBm}(21$ $\mathrm{dBm})$.

[5] ElSawy, H., Hossain, E., \& Alouini, M. S. (2014). Analytical modeling of mode selection and power control for underlay D2D communication in cellular networks. IEEE Transactions on Communications, 62(11), 4147-4161.

[6] Tong, F., Wan, Y., Zheng, L., Pan, J., \& Cai, L. (2017). A Probabilistic DistanceBased Modeling and Analysis for Cellular Networks With Underlaying Deviceto-Device Communications. IEEE Trans. Wireless Communications, 16(1), 451-463.

[7] Wang, H., \& Chu, X. (2012). Distanceconstrained resource-sharing criteria for device-to-device communications underlaying cellular networks. Electronics letters, 48(9), 528-530.

[8] Li, Y., Jiang, T., Sheng, M., \& Zhu, Y. (2016). QoS-aware admission control and resource allocation in underlay device-todevice spectrum-sharing networks. IEEE Journal on Selected Areas in Communications, 34(11), 2874-2886.

[9] Giambene, G., \& Khoa, T. A. (2017). Improving Device-to-Device communications pairing for underlay cellular networks. In Networks and Communications (EuCNC), 


\section{European Conference on (pp. 1-6). About Authors} IEEE.

[10] Giambene, G., \& Khoa, T. A. (2018). Efficiency and Fairness in the Resource Allocation to Device-to-Device Communications in LTE-A. In 2018 IEEE International Conference on Communications (ICC) (pp. 1-6). IEEE.

[11] Erceg, V., Greenstein, L. J., Tjandra, S. Y., Parkoff, S. R., Gupta, A., Kulic, B., ... \& Bianchi, R. (1999). An empirically based path loss model for wireless channels in suburban environments. IEEE Journal on selected areas in communications, 17(7), 1205-1211.

[12] Y. J. Bultitude, T. Rautiainen. (2007). "IST-4-027756 WINNER II D1.1.2 V1. 2 WINNER II channel models".
Khoa Anh TRAN received his Ph.D. degrees in Telecommunication from the University of Siena, Siena, Italy, in 2017. He became a Lecturer in Faculty of Electrical and Electronic Engineering in Ton-Duc-Thang university, HCMC, Vietnam from 2010. His current research interests include Device-to-Device communication, V2I communication and Internet of Things.

"This is an Open Access article distributed under the terms of the Creative Commons Attribution License, which permits unrestricted use, distribution, and reproduction in any medium, provided the original work is properly cited (CC BY 4.0)." 